This is the submitted version of the article:

Chikoidze E., Sartel C., Mohamed H., Madaci I., Tchelidze T., Modreanu M., Vales-Castro P., Rubio C., Arnold C., Sallet V., Dumont Y., Perez-Tomas A.. Enhancing the intrinsic p-type conductivity of the ultra-wide bandgap Ga2O3 semiconductor. J ournal of Materials Chemistry C, (2019). 7. : 10231 - . 10.1039/c9tc02910a.

Available at: https://dx.doi.org/10.1039/c9tc02910a 


\title{
Enhancing the intrinsic p-type conductivity of the ultra-wide bandgap $\mathrm{Ga}_{2} \mathrm{O}_{3}$ semiconductor
}

\author{
Ekaterine Chikoidze*a, Corinne Sartel ${ }^{a}$, Hagar Mohamed ${ }^{\text {a,b }}$, Ismail Madacia, Tamar Tchelidze ${ }^{c}$, Mircea

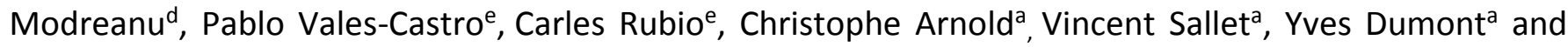 \\ Amador Perez-Tomas ${ }^{\mathrm{e}}$
}

While there are several $n$-type transparent semiconductor oxides (TSO) for optoelectronic applications (e.g. LEDs, solar cells or display TFTs), their required $p$-type counterparts oxides are known to be more challenging. For the time being, the $n$-type TSO with the largest bandgap $(\sim 5 \mathrm{eV})$ is $\mathrm{Ga}_{2} \mathrm{O}_{3}$ that holds the promisse of extending the light transparency further into the deep ultraviolet. In this work, it is demonstrated that strongly compensated $\mathrm{Ga}_{2} \mathrm{O}_{3}$ is also the intrinsic (or native) $p$-type TSO with the largest bandgap for any reported $p$-type TSO (e.g. NiO, SnO, delafossites, oxychalcogenides). The achievement of hole mobility in excess of $10 \mathrm{~cm}^{2} / \mathrm{Vs}$ and (high temperature) free hole concentrations in the $\sim 10^{17} \mathrm{~cm}^{-3}$ range challenges the current thinking about achieving $p$-type conductivity in $\mathrm{Ga}_{2} \mathrm{O}_{3}$ being "out of question". The results presented in this paper therefore further clarify that $p$-type $\mathrm{Ga}_{2} \mathrm{O}_{3}$ is possible, although more research must be conducted to determine what are the real $\mathrm{Ga}_{2} \mathrm{O}_{3}$ prospects for solar blind bipolar optoelectronics and ultra-high power electronics based in $p$ - $n$ homojunctions.

\section{Introduction}

Recently, ultra-wide band gap gallium oxide $\left(\mathrm{Ga}_{2} \mathrm{O}_{3}\right)$ is receiving a lot of renewed attention as a transparent semiconducting oxide (TSO) champion owing to its unusual material properties [1], [2], [3], large tuneable conductivity ${ }^{[4],[5]}$, extremely high breakdown field ${ }^{[6],[7],[8],}$ unique optoelectronic properties ${ }^{[9],[10],[11]}$ and low cost ${ }^{[12]}$. TSOs are a class of key enabling material in increasingly high demand because of the immediate applications they can find in a variety of new technologies, ranging from thin-film coatings and sensor devices, to transparent electronics and optoelectronics in telecommunications ${ }^{[13,14]}$. Some TSOs are indeed the standard choice for use in transparent electrode applications ${ }^{[15]}$ and are also hugely important for information and communication technologies such as displays, touch screens, solar cells or light emitting diodes ${ }^{[13]}$. Another key factor in the adoption of oxide semiconductors is that they are compatible with the strict manufacturing requirements of large-scale, largevolume, flexible, low cost and disposable/reusable devices ${ }^{[16], ~[17]}$. The arising TSO technology based in ultrawide bandgap gallium oxide $(\sim 4.8 \mathrm{eV})$ holds the promise of extending many optoelectronic applications further into the deep ultraviolet range, overpassing the conventional wide bandgap TSO's near ultraviolet limit ( 3.5 eV) (e.g. $\mathrm{ZnO}, \mathrm{SnO}_{2}$ or $\ln _{2} \mathrm{O}_{3}$ ).
However, to exploit the full potential of any emerging ultra-wide bandgap transparent optoelectronic technology, both, $n$-type and $p$-type conductivity (i.e. bipolarity), should be attained. Intrinsic wide bandgap transparent semiconductor oxides are predominately $n$ type due to their ability to form oxygen vacancies ${ }^{[18]}$. Depositing or annealing in reduced oxygen partial pressure can increase the conductivity of the oxide by introducing more carriers. In contrast, there are few predominantly $p$-type oxide semiconductors due to cation vacancies such as $\mathrm{Cu}_{2} \mathrm{O}, \mathrm{SnO}$ or $\mathrm{NiO}{ }^{[19],[20]}$. The majority of oxides have fundamental thermodynamic constrains, making their $p$-type doping difficult. More specifically, the formation of compensating intrinsic defects when lowering the Fermi energy towards the valence band has been identified as a main impediment to $p$-type doping in many oxides ${ }^{[21]}$. In any case, oxides generally exhibit an important doping asymmetry and bipolar oxide semiconductors ${ }^{[22]}$ represent only a small subset (e.g. $\mathrm{CuInO}_{2}{ }^{[23]}, \mathrm{SnO}^{[24]}, \mathrm{Ni}_{x} \mathrm{Cd}_{1-x} \mathrm{O}_{1+\delta}{ }^{[25]}, \mathrm{SnNb}_{2} \mathrm{O}_{6}$ ${ }^{[26]}$, ZrOS $\left.{ }^{[27]}\right)$. Bipolar electronic features are also extremely interesting for $\mathrm{Ga}_{2} \mathrm{O}_{3}$ ultra-wide bandgap power electronics to define the main blocking $p-n$ junctions ${ }^{[28],[29] .}$

Here, we provide stronger experimental supporting our previous report ${ }^{[30]}$ underlying that the $\mathrm{Ga}_{2} \mathrm{O}_{3}$ is a $p$-type native (or intrinsic) semiconductor. In addition, we report a route for the enhancement of the native $p$-type conductivity in undoped $\mathrm{Ga}_{2} \mathrm{O}_{3}$ by 
annealing in oxygen atmosphere. While high $n$-type conductivity in $\beta-\mathrm{Ga}_{2} \mathrm{O}_{3}$ can be efficiently achieved by impurity doping with $\mathrm{Sn}, \mathrm{Si}, \mathrm{Ge}, \mathrm{F}$ or $\mathrm{Cl}{ }^{[31,32]}$ (and even metallic conductivity due to charge accumulation on the surface in undoped $\beta-\mathrm{Ga}_{2} \mathrm{O}_{3}$. $\left.{ }^{[3,33]}\right), \quad p$-type conductivity is still controversial. Remarkably we corroborate that, thanks to the $\beta-\mathrm{Ga}_{2} \mathrm{O}_{3}$ particular point defect chemistry and the large formation energy of oxygen vacancies ${ }^{[5]}$ (the native donor which can play role of compensators for holes), it is possible the realization of a $p$-type conductivity even in undoped layers ${ }^{30}$.

\section{Results and discussion}

\section{Thermodynamic analyses}

Thermodynamic analyses of point defects and charge carriers have been carried out to determine the best conditions for growing highly compensated $p$-type $\beta$ $\mathrm{Ga}_{2} \mathrm{O}_{3}$ thin-films. By using the Kroger method of quasichemical equations ${ }^{34}$, the thermodynamic equilibrium in the $\mathrm{\beta}-\mathrm{Ga}_{2} \mathrm{O}_{3}$ (crystal) $-\mathrm{O}_{2}$ (gas) system was established to define the dependence of point defects and charge carriers on temperature $(T)$ and oxygen partial pressure $\left(P_{\text {hole }}\right)$. In general, high oxygen pressures are required to achieve $p$-type conductivity triggered by native (or intrinsic) acceptors, which are associated to gallium vacancy $\left(V_{G a}\right)$. The thermodynamic analysis show that high oxygen pressures guarantee the appearance of uncompensated hole conductivity effectively suppressing the compensation by native donors, which are associated primarily to oxygen vacancies $\left(\mathrm{V}_{0}\right)$.
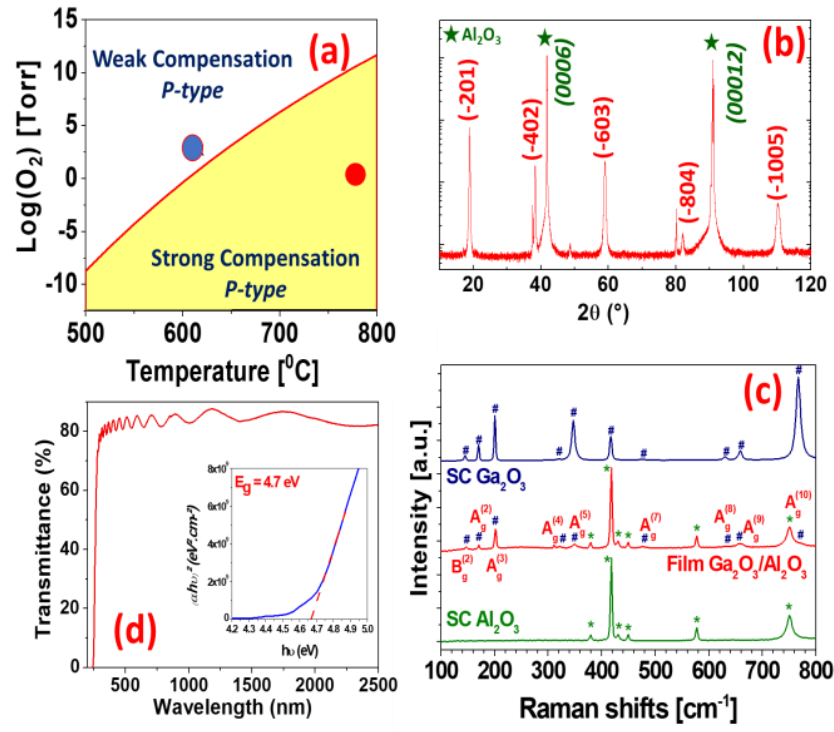

Fig. 1. (a) The dependence of growth pressure when hole conductivity appears, on growth temperature. The red point corresponds to our growth conditions, while blue point corresponds to post annealing conditions, $\mathrm{T}=600^{\circ} \mathrm{C}$, $1 \mathrm{~atm}$. Pressure. b) The $X$-ray diffractogram of $\beta-\mathrm{Ga}_{2} \mathrm{O}_{3}$ film grown on (0001) sapphire (intensity in log scale); the peaks labelled by "*" indicates the XRD reflection corresponding to the sapphire substrate; c) The Raman spectra recorded using 514.5nm excitation for: Red trace:(-201) $\beta-\mathrm{Ga}_{2} \mathrm{O}_{3}$ thin film grown on c-cut sapphire substrates ; green trace :blank c-cut sapphire Blue trace: (100) $\beta-\mathrm{Ga}_{2} \mathrm{O}_{3}$ single crystal. The phonon modes for $\beta-\mathrm{Ga}_{2} \mathrm{O}_{3}$ are labeled using "\#” while the phonon modes for $\alpha-\mathrm{Al}_{2} \mathrm{O}_{3}$ substrate are labeled using "*".

The growth conditions (oxygen partial pressure and temperature) in which acceptors become dominant species, (i.e., the compensating donor defects are suppressed), can be determined by means of the following expression:

$P_{\text {hole }}=\frac{K_{S}^{2 / 3} K_{D}^{2}}{K_{i}^{2} K_{V}^{2 / 3}}$

Where $K_{S}, K_{D}, K_{i}$ and $K_{V}$ are the reaction constants of creation of Schottky pair, ionization of native donor, lattice thermal ionization and creation of oxygen vacancy respectively. Details about the method were reported in Ref.30. As shown in the map of Fig. 1 (a), the pressure required for intrinsic $p$-type conductivity increases sharply with $T$. If the growth conditions are located in the upper part of the map, it should be expected rather uncompensated $\mathrm{Ga}_{2} \mathrm{O}_{3}$ (i.e., low $p$-type conductivity), whereas if growth conditions are located in the lower part, highly compensated samples should 
be obtained (higher $p$-type conductivity). Wang and coauthors has suggested also that the high the high temperature and high oxygen partial pressure are in favor of p-type doping in $\beta-\mathrm{Ga} 2 \mathrm{O} 3$ crystal by weakening the self-compensation effect. ${ }^{35}$

Therefore, to achieve larger intrinsic $p$-type conductivities, strongly compensated $\beta-\mathrm{Ga}_{2} \mathrm{O}_{3}$ layers should be grown, as it is demonstrated by metalorganic chemical vapour deposition (MOCVD) on sapphire substrates. Growth details are given in the experimental details section. It is worth mentioning that the $\mathrm{Ga}_{2} \mathrm{O}_{3} p$ type conductivity has been consistently measured in eight different samples (under varying growth conditions) but, in the following, the discussion is restricted for simplicity to the most illustrative one.

\section{Sample preparation and Physical Properties}

$\beta-\mathrm{Ga}_{2} \mathrm{O}_{3}$ high quality thin films have already been grown by MOCVD using commercial metalorganic precursors such as trimethyl-gallium (TMGa), and pure oxygen ${ }^{[35-38]}$. MOCVD is an industrial technique which allows the growth of large-area, uniform semiconductor layers (e.g. III-V compounds) with excellent control of the purity. In the case of $\beta-\mathrm{Ga}_{2} \mathrm{O}_{3}$, we thus expect high resistivity of the grown material due to a very low concentration of impurities. In agreement with that point, Almena et al [36] observed highly resistive electrical properties as no measurable conductivity was observed by Hall measurements, and Sbrockey el $\mathrm{al}^{37}$ reported insulating films with sheet resistivity $>10^{10} \mathrm{Ohms} / \mathrm{square}$, beyond the measurement range of their four-probe equipment. Herein, a strongly compensated undoped $\beta-\mathrm{Ga}_{2} \mathrm{O}_{3}$ layer ( $500 \mathrm{~nm}$-thick) was grown (see experimental section) on c-sapphire substrates at $T=775^{\circ} \mathrm{C}$ and $P O_{2}=5$ Torr (red dot in Fig.1(a)X-ray Cu-K $\alpha$ diffractograms, recorded between $2 \theta=10^{\circ}$ and $130^{\circ}$, in $\theta / 2 \theta$ configuration, exhibit a highly texture of gallium oxide in the $\beta-\mathrm{Ga}_{2} \mathrm{O}_{3}$ phase with monoclinic space group $(\mathrm{C} 2 / \mathrm{m})$ symmetry (Fig. $1(b))$. More intense peaks correspond to $(0,0,0,6)$ and $(0,0,0,12)$ Bragg reflections of $\mathrm{c}-\mathrm{Al}_{2} \mathrm{O}_{3}$ substrates (with also tiny peaks at $2 \theta=17.07^{\circ}$ and $37,60^{\circ}$ associated to $\mathrm{Cu}$ $\mathrm{K} \beta$ radiation). The more intense Bragg peaks of the film, at $2 \theta$ values of $18.91^{\circ}, 38.33^{\circ}, 58.98^{\circ}, 82,01^{\circ}$ and $110,21^{\circ}$, correspond to the family $(-2 n, 0, n)$ planes. Their indexes in $\mathrm{C} 2 / \mathrm{m}$ space group are $(-201),(-402),(-603),(-$ 804), (-1005) respectively, with a reticular distance $d(-$ $2,0,1)=0.4690 \mathrm{~nm}$, and intensity ratio in agreement with previous works for $\beta-\mathrm{Ga}_{2} \mathrm{O}_{3}$ films grown on $\mathrm{c}$ - sapphire ${ }^{[38-43]}$.The only additional detected Bragg peak at $2 \theta$ value of $48.71^{\circ}$ might be indexed with $\left(\begin{array}{lll}5 & 0 & 1\end{array}\right) \beta$ $\mathrm{Ga}_{2} \mathrm{O}_{3}$, with an intensity ratio lower than $0.5 \%$, and may be negligible in term of crystallized volume. So we concluded that studied thin films are textured $(-201) \beta$ $\mathrm{Ga}_{2} \mathrm{O}_{3}$ crystalline phase. The film crystalline quality has also been analysed by Raman spectroscopy. Fig. 1(c) show the Raman spectra recorded using $514.5 \mathrm{~nm}$ excitation for: in red curve $(-201)$ textured $\beta-\mathrm{Ga}_{2} \mathrm{O}_{3}$ thin film grown on c-cut sapphire substrates; in green curve a blank c-cut sapphire (0001) $\alpha-\mathrm{Al}_{2} \mathrm{O}_{3}$; and in blue trace, a (100)-cut $\beta-\mathrm{Ga}_{2} \mathrm{O}_{3}$ single crystal. The primitive unit cell of $\beta-\mathrm{Ga}_{2} \mathrm{O}_{3}$ consists of 10 atoms which results in 30 phonon modes; of which 27 are optical modes. At the $\Gamma$ -point, these belong to the irreducible representation $^{[44]}$.

$\Gamma^{\mathrm{opt}}=10 \mathrm{~A}_{\mathrm{g}}+5 \mathrm{~B}_{\mathrm{g}}+4 \mathrm{~A}_{\mathrm{u}}+8 \mathrm{~B}_{\mathrm{u}}$

The modes with $\mathrm{A}_{\mathrm{g}}$ and $\mathrm{B}_{\mathrm{g}}$ symmetry are Raman active, while those with odd parity (index $u$ ) are infrared active. The Raman spectrum for the (-201) textured $\beta-\mathrm{Ga}_{2} \mathrm{O}_{3}$ thin film grown on c-cut sapphire substrates is a combination of $\beta-\mathrm{Ga}_{2} \mathrm{O}_{3}$ (labelled using "\#" in Fig. 1(c)) and $\alpha-\mathrm{Al}_{2} \mathrm{O}_{3}$ phonon modes (labelled using "*" in Fig. 1c). No Raman phonon modes belonging to other $\mathrm{Ga}_{2} \mathrm{O}_{3}$ polymorphs have been detected thus demonstrating that we have a single $\beta-\mathrm{Ga}_{2} \mathrm{O}_{3}$ phase. The $\mathrm{Ga}_{2} \mathrm{O}_{3}$ thin film Raman phonon modes Full width at half maximum (FWHM) of the $\beta-\mathrm{Ga}_{2} \mathrm{O}_{3}$ is closed to that of single crystal $\beta-\mathrm{Ga}_{2} \mathrm{O}_{3}$ and this is indicative for a very good crystallinity. Fig. 2(d) shows transmission spectrum T[\%] in 200-2500 $\mathrm{nm}$ range. The $\mathrm{Ga}_{2} \mathrm{O}_{3}$ thin-films are highly transparent, with T 80\% in UV-VIS-NIR. The optical band gap determined as $E_{g}=4.7 \pm 0.1 \mathrm{eV}$, from classical Tauc's plot extrapolation (see the insert in Fig.2 (d)), which is also in agreement with previous reports ${ }^{[30]}$.

\section{Electrical properties}

Here, a detailed study of the electrical transport properties of strongly compensated $\beta-\mathrm{Ga}_{2} \mathrm{O}_{3}$ has been performed (while others were unsuccessful) thanks to home developed high temperature and high impedance set-up. Van der Pauw Hall Effect measurements were employed to determine the resistivity, carrier type, density and mobility. I-V characteristic for Ohmic contacts at $700 \mathrm{~K}$ is shown in insert of Figure 2(a). Resistivity at $T=530 \mathrm{~K}$ (the lowest temperature for valid measurements) was found to be $\rho=2.9 \times 10^{5} \Omega \cdot \mathrm{cm}$. By heating the sample up to $T=850 \mathrm{~K}$, the resistivity 
decreases down to $\rho=1.2 \times 10^{3} \Omega \cdot \mathrm{cm}$ as shown in Fig. 2(a). The resistivity dependence on $T-\rho(T)$, shows the typical behaviour of semi-insulating material, with a hopping conductivity enhancement above 650K (see Fig. 2 (b)). The determination of the majority carrier type is not trivial in practice when the sample is of high resistance $\left(>10^{7} \Omega\right.$ ) due to the difficulty in correctly extracting the Hall voltage $\left(V_{H}\right)$ from the total measured voltage. To further validate the sign of majority carriers, the Hall voltage dependence on the applied magnetic field was measured at different temperatures. Hall voltage measurements were carried out at varying magnetic fields (0-1.6 T) in the temperature range of $650 \mathrm{~K}-850 \mathrm{~K}$. In a non-magnetic material, $V_{H}$ is linearly proportional to the applied magnetic field and a positive sign indicates that the majority charge carriers are $p$ type (holes). As shown in Fig. 2(c), the positive $\mathrm{V}_{\mathrm{H}}$ linearly increase with perpendicularly applied magnetic field. This confirms that the layer was $p$-type. The temperature dependence for Hall hole concentration is shown in Fig. 2(d). At the largest available temperature of $850 \mathrm{~K}$, the free hole concentration is $p=5.6 \times 10^{14} \mathrm{~cm}$ ${ }^{3}$. Hall hole mobilities varies between $\mu=9.6-8.0 \mathrm{~cm}^{2} / \mathrm{Vs}$ in 680-850 $\mathrm{K}$ temperature range.
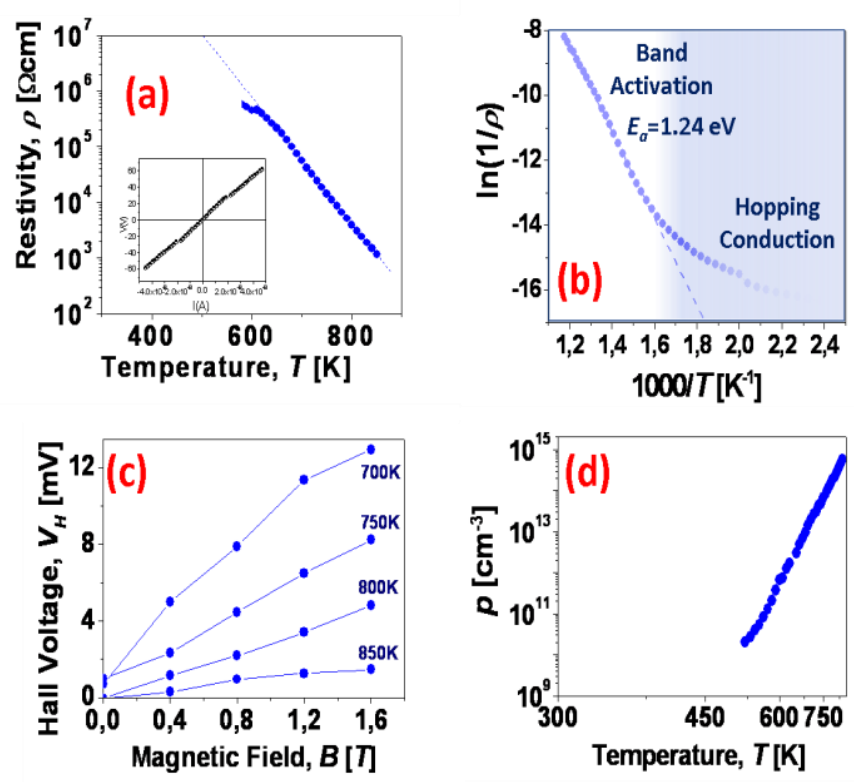

Fig.2 a) show the resistivity versus temperature change for $\mathrm{\beta}_{-}-\mathrm{Ga}_{2} \mathrm{O}_{3}$ thin films, from $\mathbf{5 3 0 - 8 5 0} \mathrm{K}$; Insert: I-V characteristics of 2-point contacts measurements at $700 \mathrm{~K}$. b) $\ln (\sigma)$ versus $T$ dependence: two regime of conductivity, band activation above $T>625 \mathrm{~K}$ and at bellow temperature when hopping conduction starts to be more important . activation energy of conductivity estimated as $E_{a}=1.24 \mathrm{eV}$ c) Hall voltage versus magnetic field at different temperatures; d)Temperature dependence for Hall hole concentration.

A carrier activation energy of $E_{a}=1.24 \mathrm{eV} \pm 0.05 \mathrm{eV}$ has been determined from the $\ln (p)$ versus $1 / T$ plot. For a $p$ type semiconductor, the general description of the hole concentration at a temperature $T(K)$ is given by the neutrality equation:

$$
\frac{g_{d} p\left(N_{D}+p\right)}{M_{v}\left(N_{A}-N_{D}-p\right)}=\exp \left(-\frac{E_{i}}{k T}\right)
$$

Where $k$ is Boltzmann's constant $\left(\mathrm{m}^{2} \mathrm{~kg} \mathrm{~s}^{-2} \mathrm{~K}^{-1}\right), g_{D}$ is the acceptor degeneracy, $N_{A}$ is the acceptor concentration $\left(\mathrm{cm}^{-3}\right)$ and $N_{D}$ is the compensating donor concentration $\left(\mathrm{cm}^{-3}\right) . M_{V}$ is the hole density of states in the valence band. If $p \ll N_{A}-N_{D}$ and $p \ll N_{D}$, in the strong compensation regime, the neutrality equation (2) becomes :

$p=\left(\frac{N_{A}-N_{D}}{N_{D}}\right)\left[\frac{M_{v}}{g_{d}}\right] \exp \left(-\frac{E_{i}}{k T}\right)$

Thus, the experimental activation energy of $E_{a}=E_{i}=1.24$ $\mathrm{eV}$ should correspond to the ionization energy of the acceptor centre $\left(E_{i}\right)$. This ionization energy value agrees well with two-times of the activation energy estimated (0.56 eV) for a previously reported arguably $p$-type weakly compensated $\mathrm{Ga}_{2} \mathrm{O}_{3}$ thin-films grown by pulsed laser deposition (PLD) ${ }^{[30]}$. A gallium vacancy acceptor level $\mathrm{V}_{\mathrm{Ga}}$ located at $1.2 \mathrm{eV}$ above the valence band has been already reported by several groups by deep-level transient spectroscopy (DLTS) ${ }^{45,46}$.

\section{Oxygen Annealing}

Annealing in an oxygen rich (i.e. Ga deficient) atmosphere should lead to an increase of acceptor $V_{G a}$ centres. As many other metal oxides, gallium oxide is a well-known material for gas sensing ${ }^{47}$ and, therefore, the effect of oxygen annealing at high temperatures on the conductivity of insulating bulk $\beta-\mathrm{Ga}_{2} \mathrm{O}_{3}$ have been intensively investigated previously ${ }^{[48,49]}$. For $\beta-\mathrm{Ga}_{2} \mathrm{O}_{3}$, it has been reported a conductivity activation temperature onset of around $\sim 700^{\circ} \mathrm{C}$. Annealing above $700^{\circ} \mathrm{C}$ in air or oxygen ambient would lead to the creation of oxygen vacancies $\left(\mathrm{V}_{\mathrm{O}}\right)$ due to the outdiffusion of oxygen atoms from the $\mathrm{Ga}_{2} \mathrm{O}_{3}$ lattice. This generation of $\mathrm{V}_{\mathrm{O}}$ leads to an additional source of freeelectrons and to a $n$-type conductivity enhancement. The ionization energy of $\mathrm{V}_{\mathrm{O}}$ in case of ceramic $\beta-\mathrm{Ga}_{2} \mathrm{O}_{3}$ 
samples has been estimated to be $\sim 1.5 \mathrm{eV}$ from the conduction band ${ }^{[50]}$. The effect of oxygen annealing leading decrease of background $n$ type carrier concentrations for diodes annealed in 02 ambient and for annealed sabstrated have been demonstrated. ${ }^{\text {[51-54] }}$.

Therefore, to enhance hole conductivity while not creating additional oxygen vacancies, post annealing in pure oxygen atmosphere at a reduced temperature of $600^{\circ}$ have been carried out in a condition of weak compensation (blue spot in Fig. 1(a)). It is worth to be mentioned that, after annealing, neither $X$-ray diffractograms nor Raman spectroscopy evidenced any change so the gallium oxide films would remain structurally unaffected. Electrical transport measurements showed that oxygen annealing leads the decrease of resistivity from $\rho=1.2 \times 10^{3} \Omega \cdot \mathrm{cm}$ to $\rho=1.7$ $\mathrm{x} 10^{2} \Omega \cdot \mathrm{cm}$ at $T=850 \mathrm{~K}$ while, at room temperature, the resistivity was determined to be $\rho=1 \times 10^{4} \Omega \cdot \mathrm{cm}$ (Fig. $3(a))$. We have not detected any "electrical inhomogeneity" performing I-V characterization and resistivity measurements, which could be in case of surface conductivity resulting bi-layered structure. A double-slope conductivity curve with two different activation energies has been determined with (high temperature) $E a_{1}=0.56 \mathrm{eV}$ and (low temperature) $E a_{2}=$ $0.17 \mathrm{eV}$ (Fig. 3(b)). In the high temperature range, the activation energy $\left(E a_{1}=0.56 \mathrm{eV}\right)$ is similar to the half of the activation energy of the same acceptor centre but in highly compensated conditions $\left(E_{i}=1.24 \mathrm{eV}\right)$. The low temperature activation energy $\left(E a_{2}\right)$ might be related with another shallow acceptor centre created after annealing in the oxygen atmosphere.

As in the as-grown samples, Hall Effect measurement methodology (measure Hall voltage with different applied magnetic field) the same as for grown sample, was applied showing that the annealed sample is $p$-type as well (See Fig. 3(c)). In this case, the Hall hole concentration was significantly increased up to $p=$ $5.6 \times 10^{17} \mathrm{~cm}^{-3}(T=850 \mathrm{~K})$, when compared with available free-hole concentration before annealing $\left(p=5.6 \times 10^{14}\right.$ $\left.\mathrm{cm}^{-3}\right)$. Nevertheless, the Hall mobility is lower, $\mu=$ $0.4 \mathrm{~cm}^{2} \mathrm{~V} / \mathrm{s}\left(\mu \sim 8.0 \mathrm{~cm}^{2} / \mathrm{Vs}\right.$ before annealing), probably due to an increased number of scattering centers. It must be mentioned that to perform Hall Effect at entire temperature range was complicated owing to this low mobility. Though still, it was actually measured in a narrower temperature range, it appears that the hole mobility does not change significantly with temperature.
Therefore, it is concluded that the conductivity activation energy is mainly determined by the activation of free carriers with temperature (Fig.3(d)). Therefore, we could assume that the conductivity activation energy of $E a_{2}=0.17 \mathrm{eV}$ is the acceptor level energy with an ionization energy of around this value.
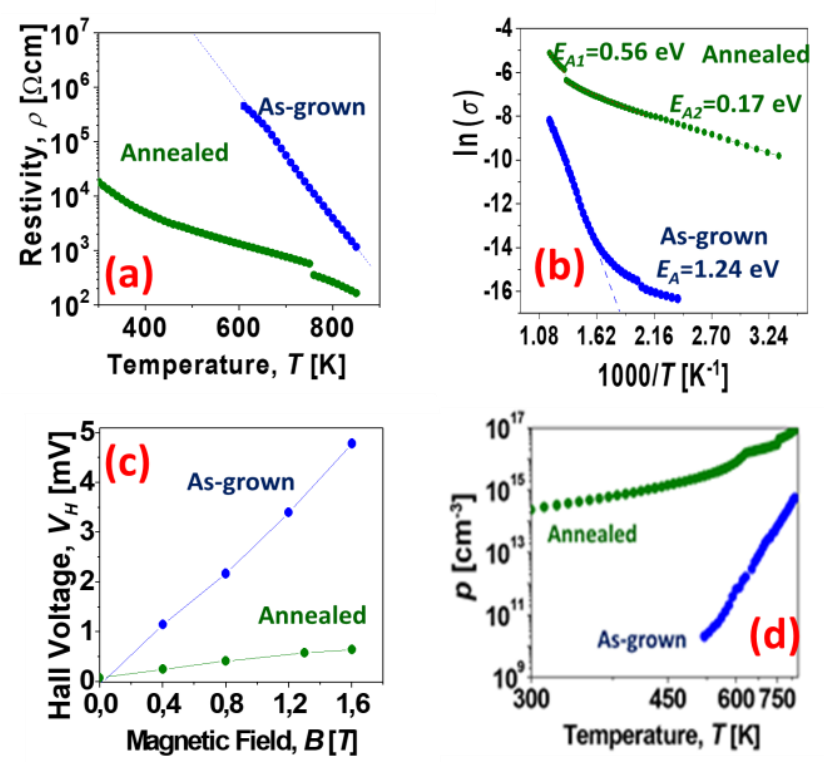

Fig.3 a) Resistivity temperature dependence $b) \ln (\sigma)$ versus $\mathrm{T}$ dependence $\mathrm{c}$ )Hall voltage $\mathrm{VH}$ versus applied external magnetic field at $\mathrm{T}=850 \mathrm{~K}$ d) Hall hole concentrations versus temperature for as grown and annealed in $\mathrm{O}_{2}$ at $600^{\circ} \mathrm{C} \beta$ $\mathrm{Ga}_{2} \mathrm{O}_{3}$ thin films.

A potential origin of this shallow level may be further elucidated by considering several theoretical assumptions. It is well-known that, close to the stoichiometry, oxide's oxygen vacancies tend to be preferentially in a double charged $\left(\mathrm{V}_{\mathrm{O}}^{++}\right)$state then as single charge $\left(\mathrm{V}_{\mathrm{O}}^{+}\right)$state ${ }^{[55]}$. In off-stoichiometry state, when hole concentration are important, the compensating donor defects are expected to be even more abundant in double charged state. Taking this fact into account, we attribute the shallower acceptor centre with $E a_{2}=0.17 \mathrm{eV}$ activation energy to be due to the creation of a $\mathrm{V}_{\mathrm{Ga}}{ }^{-}-\mathrm{V}_{\mathrm{O}}{ }^{++}$complexes. Indeed, the high probability of creation of such vacancy complexes has been studied in detail by Ingebrigtsen et al ${ }^{[56]}$. In the case of oxygen annealed strongly compensated $\beta-\mathrm{Ga}_{2} \mathrm{O}_{3}$, because of the Coulombic interactions between $\mathrm{V}_{\mathrm{Ga}}{ }^{-}$and $\mathrm{V}_{\mathrm{O}}{ }^{++}$, the $\mathrm{V}_{\mathrm{O}}^{++}$donor centre has a tendency to be located in the vicinity of charged acceptor centre, forming $\mathrm{V}_{\mathrm{Ga}}$ $\mathrm{V}_{\mathrm{O}}{ }^{++}$complexes. Consequently, the energy of electron localized on $\mathrm{V}_{\mathrm{Ga}}$ ' decreased by amount of $\Delta \boldsymbol{E}=$ 
$2 \boldsymbol{e}^{2} / \boldsymbol{\varepsilon} \boldsymbol{R}_{A s}$, where $\boldsymbol{R}_{A s}$ is distance between $\mathrm{V}_{\mathrm{Ga}}{ }^{-}$and $\mathrm{V}_{\mathrm{O}}{ }^{++}, \varepsilon$ is static dielectric constant of $\mathrm{Ga}_{2} \mathrm{O}_{3}$. If $\mathrm{V}_{0}$ " defect is located in the first coordination spheres, its proximity can reduce electronic level of $\mathrm{V}_{\mathrm{Ga}}{ }^{\prime}(1.24 \mathrm{eV})$ by $\sim 1 \mathrm{eV}$, which is in a good agreement with $E a_{2}=0.17 \mathrm{eV}$ activation energy observed in experiment.

\section{Chemical analysis: XPS $p$-type valence band}
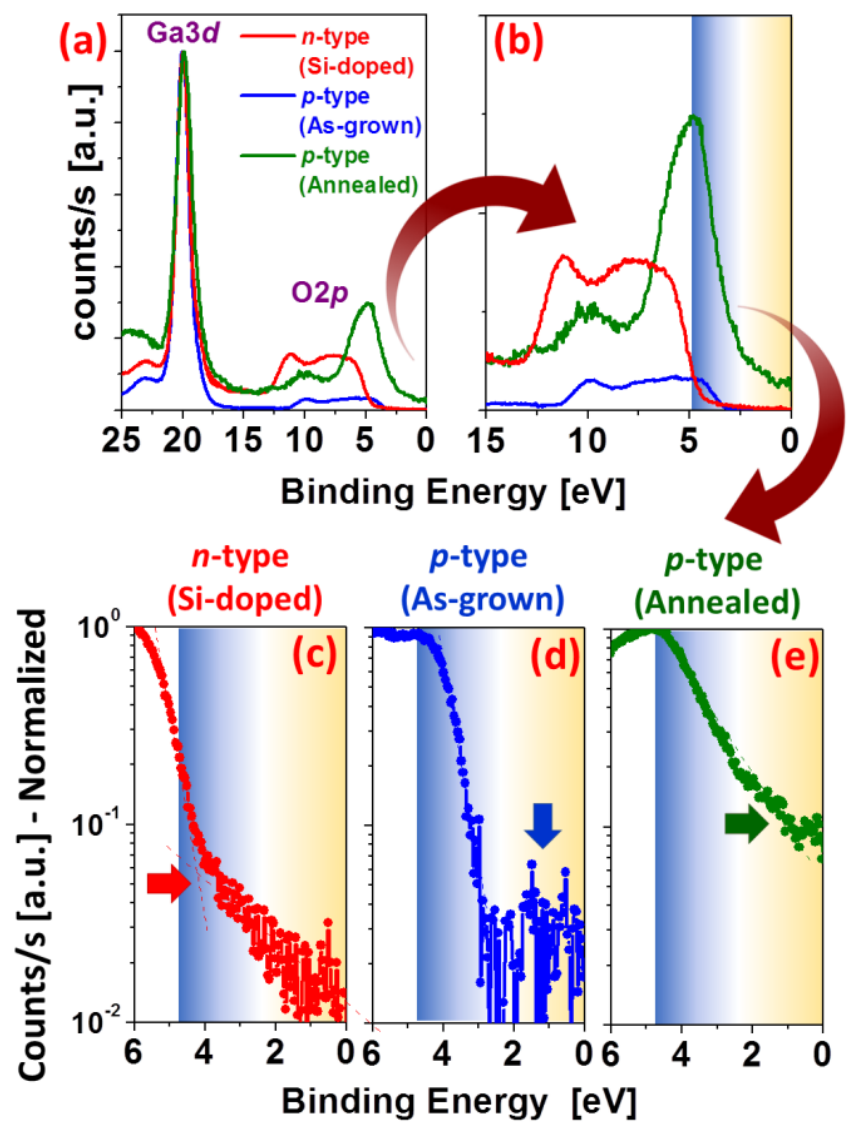

Fig.4 (a) X-ray photoelectron spectroscopy of the $\mathrm{Ga}_{2} \mathrm{O}_{3}$ valence band for a commercial Si-doped ( $n$-type) reference and the $p$-type as-grown/annealed of this work (calibrated with respect to Ga3d). (b) A zoom of the valence band region, which in the case of stoichiometric $\mathrm{Ga}_{2} \mathrm{O}_{3}$, has a $\mathrm{O} 2 p$ character. Investigation of the valence band tail states for (c) $n$-type (Si-doped), (d) $p$-type (as-grown) and (e) $p$-type (annealed) evidencing the presence of states in the lower part of the bandgap.
X-ray photoelectron spectroscopy (XPS) is a fine chemical method to investigate a material's valence band characteristic and brings additional evidence of the presence of states within the bandgap ${ }^{57-59}{ }^{57}$ When used in high resolution in the valence band vicinity (i.e., for the lowest binding energies), it is possible to directly detect whether there are states in the lower part of the bandgap (those responsible of the $p$-type character) ${ }^{60}$

As shown in Fig. 4, it was found a clear correlation within the amount of states in the lower half of the bandgap and the $p$-type character in the sequence, $n$-type (Sidoped) commercial reference, $p$-type (undoped) and $p$ type (annealed). The $n$-type control sample is a commercial (Novel Crystal Technology, Inc.) nominally $n$-type Si-doped $\beta-\mathrm{Ga}_{2} \mathrm{O}_{3}\left(\mathrm{~N}_{\mathrm{D}}-\mathrm{N}_{\mathrm{A}}=1.3 \times 10^{18} \mathrm{~cm}^{-3}\right)$ epitaxy $(500 \mathrm{~nm})$ grown on a single crystal $\beta-\mathrm{Ga}_{2} \mathrm{O}_{3}$ (labelled " $n$-type (Si-doped)"). For our samples, the valence band edge is shifted towards more $p$-type using the Ga3d peak as the calibration peak (smaller Fermi level implies more $p$-type character), see Fig. 4(a) and (b). The numerical values of the energy levels are in agreement with those given by Hall. A zoom of the smallest binding energies further reveals the presence of states within the bandgap (Fig. 4(c),(d) and (e)). The counts per second (counts/s) are normalized to the maximum value in the range of $0-6 \mathrm{eV}$. The Fermi level is near the conduction band in the case of the commercial Si-doped $\mathrm{Ga}_{2} \mathrm{O}_{3}$ reference $(\sim 4.4 \mathrm{eV})$. For the $\mathrm{Ga}_{2} \mathrm{O}_{3}$ reference, there is no indication of further tail states within the bandgap. The Fermi level is shifted towards an intrinsic value (i.e., mid-gap) in our undoped as-grown $\mathrm{Ga}_{2} \mathrm{O}_{3}$, but a number of tail states arise at $\sim 1.5$ $1.2 \mathrm{eV}$ from the conduction band. In the annealed sample, the states further extend towards the lower part of the bandgap and hence, indicating a much larger $p$-type carrier density (and hence, conductivity), as the I$V$ and Hall experiments suggest. From the extrapolation of the valence band edge, the Fermi level is as low as $\sim 0.2 \mathrm{eV}$ for the annealed sample.

\section{Comparison with the state-of-the-art}

In any case, for the majority of oxides, to achieve sizable $p$-type conductivity is challenging because their valence band maximum consists of strongly localized $02 p$ derived orbitals. In other words, the valence band of most oxides is rather flat, resulting in deep acceptor levels (lower free hole concentration at room temperature) and larger hole effective masses (lower 
mobilities) [61-62], During many years the possibility of doping $p$-type $\mathrm{Ga}_{2} \mathrm{O}_{3}$ has been considered "out of question" due to the particular flatness of the computed valence band resulting in a high hole effective mass (see e.g. ${ }^{[63]}$ ). $\mathrm{A} \mathrm{Ga}_{2} \mathrm{O}_{3}$ valence band being governed by $\mathrm{O} 2 \mathrm{p}$ orbitals with arguably very low hole mobility and having very small dispersion in the wave vector-energy space has been routinely reported ${ }^{[5], ~[66-67] . ~ I n ~ a d d i t i o n, ~ a ~ s t r o n g ~}$ localization of holes at specific sites in bulk $\mathrm{Ga}_{2} \mathrm{O}_{3}$ by $\mathrm{a}$ self-trapping effect, has also been predicted by firstprinciples calculations [68]
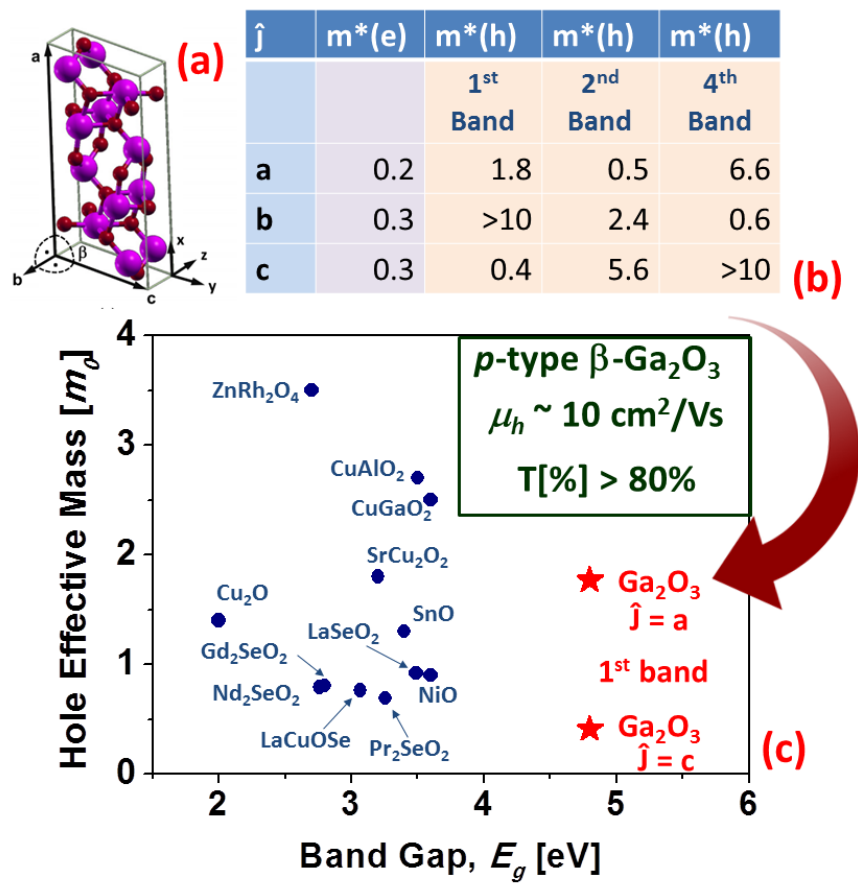

Fig. 5. a) Unit cell of $\beta-\mathrm{Ga}_{2} \mathrm{O}_{3}$ detailing crystallographic coordinate systems. b) $\mathrm{B}_{-} \mathrm{Ga}_{2} \mathrm{O}_{3}$ effective mass parameters for conduction and valence bands as indexed for lowest transition along directions $\mathrm{a}, \mathrm{b}$ and $\mathrm{c}$ (in units of $\mathrm{mo}_{\mathrm{o}}$. c) Comparison of the predicted effective mass of the ultra-wide bandgap $\mathrm{\beta}-\mathrm{Ga}_{2} \mathrm{O}_{3}$ compared with other wide-bandgap oxides [62], [63], [70], 71] . The figures a) and b) here are reproduced or adapted with permission from A. Mock et al., Band-to-band transitions, selection rules, effective mass, and excitonic contributions in monoclinic $\beta-\mathrm{Ga}_{2} \mathrm{O}_{3}$, Phys. Rev. B 96, 245205 (2017) ${ }^{69]}$ @2017 American Physical Society.

Nevertheless, it has been also recently reported that the hole conduction mass in $\mathrm{Ga}_{2} \mathrm{O}_{3}$ is, indeed, fairly anisotropic and can be as low as $0.4 m_{0}$ for certain orientations (while larger than $10 m_{0}$ in others) ${ }^{[69]}$. Fig.5 $(a, b)$.Thus, challenging current thinking, we have found experimentally a sizable hole mobility of at least $\sim 10$ $\mathrm{cm}^{2} / \mathrm{Vs}$ together with an outstanding transparency in the visible of above $80 \%$. What is more important is the significant "shift of the native $p$-type conductivity" up to the $\sim 5 \mathrm{eV}$ region ( $250 \mathrm{~nm}$ ) when compared with previous $p$-type conductive oxides such as $\mathrm{NiO}, \mathrm{SnO}, \mathrm{Cu}$ based delafossites $\left(\mathrm{CuAlO}_{2}, \mathrm{CuGaO}_{2}, \mathrm{SrCu}_{2} \mathrm{O}_{2}\right)$ or oxychalcogenides, all of them lying in the range of $\sim 2.5$ $3.7 \mathrm{eV}[62-63]$, , [70],[62-72] 72 .

\section{Conclusions}

In this work, we corroborate, via electrical Hall effect and chemical XPS measurements, that the ultra-wide band gap $\mathrm{Ga}_{2} \mathrm{O}_{3}$ oxide is an "native" $p$ type semiconductor oxide even in the case of strong compensation by donor defects. This unusual native $p$-type nature is due to the $\mathrm{Ga}_{2} \mathrm{O}_{3}$ particular point defects chemistry, mainly the large formation energy of oxygen donor vacancies, allowing the realization of a $p$-type. This confirms, in practice, that $\mathrm{Ga}_{2} \mathrm{O}_{3}$ is the $p$-type TSO with the largest band gap and also is potentially bipolar. The corroboration of this fact might have deep implications in optoelectronic, thanks to the shifting the optical band gap onset, and in power electronics in regard of the device's breakdown voltage range. Notably, the $p$-type nature was determined in samples grown by MOCVD, differently to the only previously reported native $p$ type conductivity for the samples grown by PLD [30]. Such reproducibility reinforces our claim of possible realization of native hole conductivity in Gallium Oxide. Annealing in oxygen results in much improved (three-orders of magnitude) free hole concentration $p(O x$-anneal $)=5.6 \times 10^{17} \mathrm{~cm}^{-3} \mathrm{vs}$. $p$ (as-grown $\left.)=5.6 \times 10^{14} \mathrm{~cm}^{-3}\right)(T=850 \mathrm{~K})$ and resistivity $\rho$ (Ox-anneal) $=1.7 \Omega \cdot \mathrm{cm}$ vs. $\rho$ (as-grown) $=\rho=1.2 \times 10^{3} \Omega \cdot \mathrm{cm}(T=850 \mathrm{~K})$. However, the mobility $\mu=0.4 \mathrm{~cm}^{2} \mathrm{~V} / \mathrm{s}\left(\mu \sim 8.0 \mathrm{~cm}^{2} / \mathrm{Vs}\right.$ before annealing) is reduced probably due to a greater number of acceptor scattering centres. The origin of such improved $p$-type conductivity is preliminary ascribed to the formation of $\mathrm{V}_{\mathrm{Ga}}{ }^{-}-\mathrm{V}_{\mathrm{O}}{ }^{++}$complexes, as a shallow acceptor centre.

\section{Experimental Details}


$\mathrm{Ga}_{2} \mathrm{O}_{3}$ growth details. Undoped $\beta-\mathrm{Ga}_{2} \mathrm{O}_{3}$ sample was grown in a RF-heated horizontal MOCVD reactor with separate inlets to avoid premature reactions in the manifold between oxygen and organometallics precursors. The reactor can operate at low pressure, between 30 and 760 torr, and at high growth temperature, up to $1000^{\circ} \mathrm{C}$. Trimethylgallium (TMGa) and $5.5 \mathrm{~N}$ pure oxygen were used as gallium and oxygen sources, respectively. Argon was used as carrier gas. The TMGa bubbler temperature was fixed at $-15^{\circ} \mathrm{C}$ in order to obtain a low vapour pressure, and consequently a moderate growth rate near $5 \mathrm{~nm} / \mathrm{min}$. The $\beta-\mathrm{Ga}_{2} \mathrm{O}_{3}$ layer was grown on $\mathrm{C}$-oriented sapphire substrate using $\mathrm{Ga} / \mathrm{O}$ ratio, growth temperature and reactor pressure set at $1.4 \times 10^{-4}, 775^{\circ} \mathrm{C}$, and 30 torr respectively. The layer's thickness was measured at $450 \mathrm{~nm}$.

$X$-ray diffraction profiles were recorded in $\theta / 2 \theta$ configuration, using Rigaku SmartLab equipped with $\mathrm{Cu}$ K $\alpha 1$ source $(\lambda=0,1541 \mathrm{~nm})$. Optical transmission spectra were measured in 200-2000 nm spectral range with a Perklin Elmer 9 spectrophotometer.

Raman spectra were recorded using a Renishaw Invia Reflex micro-Raman spectrometer at room temperature. The samples were excited using a cw Modu-Laser Stellar-REN laser emitting at $514.5 \mathrm{~nm}$ with a power of $2-4 \mathrm{~mW}$. The reflecting microscope objective was 50X with a NA 0.75 ; the excitation spot diameter was $1 \mu \mathrm{m}$. The back-scattered light was dispersed by a monochromator with a spectral resolution of $1.4 \mathrm{~cm}^{-1}$. The light was detected by a charge coupled device. The typical accumulation time was 30s. Raman shifts were calibrated using an optical phonon frequency $(520.5 \mathrm{~cm}$ $\left.{ }^{1}\right)$ of silicon monocrystal. Several reference Raman spectra for beta- $\mathrm{Ga}_{2} \mathrm{O}_{3}$ single crystal substrates and sapphire c-cut substrates have been also measured.

Hall Effect measurement set-up. Ohmic contacts were prepared by silver paint at the four corners of the sample. Hall Effect measurements were performed in a Van der Pauw configuration in the temperature range of $300 \mathrm{~K}$ to $850 \mathrm{~K}$ and for magnetic fields perpendicular to the film plane varying from $-1.6 \mathrm{~T}$ to $1.6 \mathrm{~T}$, using a high impedance measurement set-up which was custom designed for measurement of high resistance.

$X$-ray photoemission spectroscopy (XPS) measurements were performed with a Phoibos 150 analyzer (SPECS $\mathrm{GmbH}$, Berlin, Germany) in ultra-high vacuum conditions (base pressure $3 \times 10^{-10} \mathrm{mbar}$ ). XPS measurements were performed with a monochromatic Al KaX-ray source (1486.74 eV).

\section{Acknowledgements}

Hagar Mohammed would like to acknowledge Cultural Affairs and Massion Sector, Egyptian Ministry for Higher Education for her fellowship giving possibility work in France. We express our thanks to Guillaume Bouchez (GEMaC) for his assistance for optical measurements. APT acknowledges Agencia Estatal de Investigación (AEI) and Fondo Europeo de Desarrollo Regional (FEDER) under contract ENE2015-74275-JIN. The ICN2 is funded by the CERCA programme / Generalitat de Catalunya and by the Severo Ochoa Centres of Excellence programme, funded by the Spanish Research Agency (AEI, grant no. SEV-2017-0706).

\section{References:}

1. Nagarajan, L.; De Souza, R. A.; Samuelis, D.; Valov, I.; Börger, A.; Janek, J.; Becker, K.-D.; Schmidt, P. C.; Martin, M. A Chemically Driven InsulatorMetal Transition in Non-Stoichiometric and Amorphous Gallium Oxide. Nature Mater 2008, 7 391-398. https://doi.org/10.1038/nmat2164.

2. Kim, J.; Sekiya, T.; Miyokawa, N.; Watanabe, N.; Kimoto, K.; Ide, K.; Toda, Y.; Ueda, S.; Ohashi, N.; Hiramatsu, H.; et al. Conversion of an UltraWide Bandgap Amorphous Oxide Insulator to a Semiconductor. NPG Asia Materials 2017, 9 (3), e359. https://doi.org/10.1038/am.2017.20.

3. Chikoidze, E.; Rogers, D.; H. Teherani, F.; Ton, C.; Tchelidze, T.; Dumont, Y.; Perez-Tomas, A. Puzzling Robust 2D Metallic Conductivity in Undoped B-Ga2O3 Thin Films. 2019, 8, 10. https://doi.org/10.1016/j.mtphys.2018.11.006.

4. Orita, M.; Ohta, H.; Hirano, M.; Hosono, H. DeepUltraviolet Transparent Conductive $\beta-\mathrm{Ga} 2 \mathrm{O} 3$ Thin Films. Applied Physics Letters 2000, 77, 4166-4168. https://doi.org/10.1063/1.1330559.

5. Varley, J. B.; Weber, J. R.; Janotti, A.; Van de Walle, C. G. Oxygen Vacancies and Donor Impurities in $\beta$-Ga2O3. Appl. Phys. Lett. 2010, 97 (14),

142106. 
https://doi.org/10.1063/1.3499306.

6. Higashiwaki, M.; Sasaki, K.; Kamimura, T.; Hoi Wong, M.; Krishnamurthy, D.; Kuramata, A.; Masui, T.; Yamakoshi, S. Depletion-Mode Ga2O3 Metal-Oxide-Semiconductor Field-Effect Transistors on $\beta$-Ga2O3 (010) Substrates and Temperature Dependence of Their Device Characteristics. Appl. Phys. Lett. 2013, 103 (12), 123511. https://doi.org/10.1063/1.4821858.

7. Higashiwaki, M.; Sasaki, K.; Murakami, H.; Kumagai, Y.; Koukitu, A.; Kuramata, A.; Masui, T.; Yamakoshi, S. Recent Progress in Ga2O3power Devices. Semicond. Sci. Technol. 2016, $31 \quad$ (3), 034001. https://doi.org/10.1088/02681242/31/3/034001.

8. Yang, J.; Ren, F.; Tadjer, M.; Pearton, S. J.; Kuramata, A. 2300V Reverse Breakdown Voltage Ga2O3 Schottky Rectifiers. ECS J. Solid State Sci. Technol. 2018, 7 (5), Q92-Q96. https://doi.org/10.1149/2.0241805jss.

9. Oshima, T.; Okuno, T.; Fujita, S. Ga2O3 Thin Film Growth on C-Plane Sapphire Substrates by Molecular Beam Epitaxy for Deep-Ultraviolet Photodetectors. Jpn. J. Appl. Phys. 2007, 46 (11R), 7217. https://doi.org/10.1143/JJAP.46.7217.

10. Lee, Y. S.; Chua, D.; Brandt, R. E.; Siah, S. C.; Li, J. V.; Mailoa, J. P.; Lee, S. W.; Gordon, R. G.; Buonassisi, T. Atomic Layer Deposited Gallium Oxide Buffer Layer Enables 1.2 v Open-Circuit Voltage in Cuprous Oxide Solar Cells. ADVANCED MATERIALS 2014, 26 (27), 4704-4710. https://doi.org/10.1002/adma.201401054.

11. Li, C.; Hisatomi, T.; Watanabe, O.; Nakabayashi, M.; Shibata, N.; Domen, K.; Delaunay, J.-J. Positive Onset Potential and Stability of Cu2OBased Photocathodes in Water Splitting by Atomic Layer Deposition of a Ga2O3 Buffer Layer. Energy Environ. Sci. 2015, 8 (5), 14931500. https://doi.org/10.1039/C5EE00250H.

12. Reese, S. B.; Remo, T.; Green, J.; Zakutayev, A. How Much Will Gallium Oxide Power Electronics Cost? Joule 2019, 3 (4), 903-907. https://doi.org/10.1016/j.joule.2019.01.011.

13. Yu, X.; Marks, T. J.; Facchetti, A. Metal Oxides for Optoelectronic Applications. Nat Mater 2016, $15 \quad$ (4), 383-396. https://doi.org/10.1038/nmat4599.

14. Ellmer, K. Past Achievements and Future Challenges in the Development of Optically
Transparent Electrodes. Nature Photonics 2012, 6 (12), 809-817. https://doi.org/10.1038/nphoton.2012.282.

15. Handbook of Transparent Conductors; Ginley, D. S., Ed.; Springer: New York, 2010.

16. Handbook of Photovoltaic Science and Engineering, 2. ed., [fully rev. and updated].; Luque López, A., Hegedus, S., Eds.; Wiley: Chichester, 2011.

17. Pasquarelli, R. M.; Ginley, D. S.; O'Hayre, R. Solution Processing of Transparent Conductors: From Flask to Film. Chem. Soc. Rev. 2011, 40 (11), 5406-5441. https://doi.org/10.1039/C1CS15065K.

18. Springer Handbook of Electronic and Photonic Materials, 2nd ed.; Kasap, S., Capper, P., Eds.; Springer Handbooks; Springer International Publishing, 2017.

19. Wang, Z.; Nayak, P. K.; Caraveo-Frescas, J. A.; Alshareef, H. N. Recent Developments in P-Type Oxide Semiconductor Materials and Devices. Adv. Mater. Weinheim 2016, 28 (20), 38313892.

https://doi.org/10.1002/adma.201503080.

20. Liu, A.; Zhu, H.; Noh, Y.-Y. Solution-Processed Inorganic p-Channel Transistors: Recent Advances and Perspectives. Materials Science and Engineering $R$ Reports 2019, 135, 85. https://doi.org/10.1016/j.mser.2018.11.001.

21. Hautier, G.; Miglio, A.; Ceder, G.; Rignanese, G.M.; Gonze, X. Identification and Design Principles of Low Hole Effective Mass $p$-Type Transparent Conducting Oxides. Nature Communications 2013, 4, 2292. https://doi.org/10.1038/ncomms3292.

22. Grundmann, M.; Klüpfel, F.; Karsthof, R.; Schlupp, P.; Schein, F.-L.; Splith, D.; Yang, C.; Bitter, S.; Wenckstern, H. von. Oxide Bipolar Electronics: Materials, Devices and Circuits. J. Phys. D: Appl. Phys. 2016, 49 (21), 213001. https://doi.org/10.1088/00223727/49/21/213001.

23. Yanagi, H.; Ueda, K.; Ohta, H.; Orita, M.; Hirano, M.; Hosono, H. Fabrication of All Oxide Transparent $\mathrm{P}-\mathrm{N}$ Homojunction Using Bipolar CulnO2 Semiconducting Oxide with Delafossite Structure. Solid State Communications 2001, 121, 15-17. https://doi.org/10.1016/S00381098(01)00439-2.

24. Graužinytė, M.; Goedecker, S.; Flores-Livas, J. A. Towards Bipolar Tin Monoxide: Revealing 
Unexplored Dopants. Phys. Rev. Materials 2018, $2 \quad$ (10), 104604. https://doi.org/10.1103/PhysRevMaterials.2.10 4604.

25. Liu, C. P.; Egbo, K. O.; Ho, C. Y.; Zapien, J. A.; Walukiewicz, W.; Yu, K. M. Stoichiometry Controlled Bipolar Conductivity in Nanocrystalline NixCd1-xO1+ $\delta$ Thin Films. Phys. Rev. Applied 2019, 11 (1), 014019. https://doi.org/10.1103/PhysRevApplied.11.01 4019.

26. Samizo, A.; Kikuchi, N.; Aiura, Y.; Nishio, K.; Mibu, K. Carrier Generation in P-Type Wide-Gap Oxide: SnNb2O6 Foordite. Chem. Mater. 2018, $30 \quad$ (22), 8221-8225 https://doi.org/10.1021/acs.chemmater.8b034 08.

27. Arai, T.; limura, S.; Kim, J.; Toda, Y.; Ueda, S.; Hosono, H. Chemical Design and Example of Transparent Bipolar Semiconductors. J. Am. Chem. Soc. 2017, 139 (47), 17175-17180. https://doi.org/10.1021/jacs.7b09806.

28. Pérez-Tomás, A.; Chikoidze, E.; Jennings, M. R.; Russell, S. A. O.; Teherani, F. H.; Bove, P.; Sandana, E. V.; Rogers, D. J. Wide and UltraWide Bandgap Oxides: Where Paradigm-Shift Photovoltaics Meets Transparent Power Electronics. In Oxide-based Materials and Devices IX; International Society for Optics and Photonics, 2018; Vol. 10533, p 105331Q. https://doi.org/10.1117/12.2302576.

29. Baliga, B. J. Fundamentals of Power Semiconductor Devices; Springer Science \& Business Media, 2010.

30. Chikoidze, E.; Fellous, A.; Perez-Tomas, A.; Sauthier, G.; Tchelidze, T.; Ton-That, C.; Huynh, T. T.; Phillips, M.; Russell, S.; Jennings, M.; et al. P-Type $\beta$-Gallium Oxide: A New Perspective for Power and Optoelectronic Devices. Materials Today Physics 2017, 3, 118-126. https://doi.org/10.1016/j.mtphys.2017.10.002.

31. Matsuzaki, K.; Hiramatsu, H.; Nomura, K.; Yanagi, H.; Kamiya, T.; Hirano, M.; Hosono, H. Growth, Structure and Carrier Transport Properties of GaO Epitaxial Film Examined for Transparent Field-Effect Transistor. Thin Solid Films 2006, 496, 37-41. https://doi.org/10.1016/j.tsf.2005.08.187.

32. Chikoidze, E.; von Bardeleben, H. J.; Akaiwa, K.; Shigematsu, E.; Kaneko, K.; Fujita, S.; Dumont, Y. Electrical, Optical, and Magnetic Properties of
Sn Doped $\alpha-\mathrm{Ga} 2 \mathrm{O} 3$ Thin Films. Journal of Applied Physics 2016, 120, 025109. https://doi.org/10.1063/1.4958860.

33. J. E. N. Swallow, J. B. Varley, L. A. H. Jones, J. T. Gibbon, L. F. J. Piper, V. R. Dhanak, and T. D. Veal, APL Materials, 7, 022528 (2019) https://doi.org/10.1063/1.5054091

34. Schmalzried, H. F. A. Kröger: The Chemistry of Imperfect Crystals, North-Holland Publishing Company-Amsterdam 1964. 1039 Seiten. Preis: Hfl. 110,-. Berichte der Bunsengesellschaft für physikalische Chemie 1964, 68 (6), 608-608. https://doi.org/10.1002/bbpc.19640680615.

35. Alema, F.; Hertog, B.; Osinsky, A.; Mukhopadhyay, P.; Toporkov, M.; Schoenfeld, W. V. Fast Growth Rate of Epitaxial $\beta-\mathrm{Ga} 2 \mathrm{O} 3$ by Close Coupled Showerhead MOCVD. Journal of Crystal Growth 2017, 475, 77-82. https://doi.org/10.1016/j.jcrysgro.2017.06.001.

36. Sbrockey, N. M.; Salagaj, T.; Coleman, E.; Tompa, G. S.; Moon, Y.; Kim, M. S. Large-Area MOCVD Growth of Ga2O3 in a Rotating Disc Reactor. Journal of Elec Materi 2015, 44 (5), 1357-1360. https://doi.org/10.1007/s11664014-3566-7.

37. Du, X.; Mi, W.; Luan, C.; Li, Z.; Xia, C.; Ma, J. Characterization of Homoepitaxial $\beta-G a 2 O 3$ Films Prepared by Metal-Organic Chemical Vapor Deposition. Journal of Crystal Growth 2014, 404, 75-79. https://doi.org/10.1016/j.jcrysgro.2014.07.011. 38. Hu, D.; Zhuang, S.; Ma, Z.; Dong, X.; Du, G.; Zhang, B.; Zhang, Y.; Yin, J. Study on the Optical Properties of $\beta-G a 2 O 3$ Films Grown by MOCVD. J Mater Sci: Mater Electron 2017, 28 (15), 10997-11001. https://doi.org/10.1007/s10854017-6882-x.

39. Oda, M.; Tokuda, R.; Kambara, H.; Tanikawa, T.; Sasaki, T.; Hitora, T. Schottky Barrier Diodes of Corundum-Structured Gallium Oxide Showing on-Resistance of $0.1 \mathrm{M} \Omega \cdot \mathrm{cm} 2$ Grown by MIST EPITAXY ${ }^{\circledR}$. Appl. Phys. Express 2016, 9 (2), 021101.

https://doi.org/10.7567/APEX.9.021101.

40. Zhong, M.; Wei, Z.; Meng, X.; Wu, F.; Li, J. HighPerformance Single Crystalline UV Photodetectors of $\beta-G a 203$. Journal of Alloys and Compounds 2015, 619, 572-575. https://doi.org/10.1016/j.jallcom.2014.09.070.

41. N. M. Sbrockley, T. Salagaj, E. Coleman, G. S. Tompa, Y. Moon, and M. Sik Kim, J. of Elec. Mat. 44, 1 (2015) 
42. Seiler, W.; Selmane, M.; Abdelouhadi, K.; Perrière, J. Epitaxial Growth of Gallium Oxide Films on C-Cut Sapphire Substrate. Thin Solid Films 2015, 589, 556-562. https://doi.org/10.1016/j.tsf.2015.06.034.

43. Chen, Y.; Liang, H.; Xia, X.; Tao, P.; Shen, R.; Liu, Y.; Feng, Y.; Zheng, Y.; Li, X.; Du, G. The Lattice Distortion of $\beta$-Ga2O3 Film Grown on c-Plane Sapphire. J Mater Sci: Mater Electron 2015, 26 (5), 3231-3235. https://doi.org/10.1007/s10854-015-2821-x.

44. Dohy, D.; Lucazeau, G.; Revcolevschi, A. Raman Spectra and Valence Force Field of SingleCrystalline $\beta$ Ga2O3. Journal of Solid State Chemistry 1982, 45 (2), 180-192. https://doi.org/10.1016/0022-4596(82)902742.

45. Polyakov, A. Y.; Smirnov, N. B.; Shchemerov, I. V.; Yakimov, E. B.; Yang, J.; Ren, F.; Yang, G.; Kim, J.; Kuramata, A.; Pearton, S. J. Point Defect Induced Degradation of Electrical Properties of Ga2O3 by $10 \mathrm{MeV}$ Proton Damage. Appl. Phys.

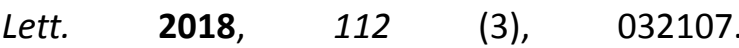
https://doi.org/10.1063/1.5012993.

46. Zhang, Z.; Farzana, E.; Arehart, A. R.; Ringel, S. A. Deep Level Defects throughout the Bandgap of (010) $\beta-G a 203$ Detected by Optically and Thermally Stimulated Defect Spectroscopy. Appl. Phys. Lett. 2016, 108 (5), 052105. https://doi.org/10.1063/1.4941429.

47. Pearton, S. J.; Yang, J.; Cary, P. H.; Ren, F.; Kim, J.; Tadjer, M. J.; Mastro, M. A. A Review of Ga2O3 Materials, Processing, and Devices. Applied Physics Reviews 2018, 5 (1), 011301. https://doi.org/10.1063/1.5006941.

48. Fleischer, M.; Meixner, H. Sensing Reducing Gases at High Temperatures Using Long-Term Stable Ga2O3 Thin Films. Sensors and Actuators $B$ : Chemical 1992, 6 (1), 257-261. https://doi.org/10.1016/0925-4005(92)800656.

49. Fleischer, M.; Meixner, H. Electron Mobility in Single- and Polycrystalline Ga2O3. Journal of Applied Physics 1993, 74 (1), 300-305. https://doi.org/10.1063/1.354107.

50. Kosacki, I.; Anderson, H. U. Nanostructured Oxide Thin Films for Gas Sensors. Sensors and Actuators B: Chemical 1998, 48 (1), 263-269. https://doi.org/10.1016/S0925-4005(98)000550 .

51. Zacherle, T.; Schmidt, P. C.; Martin, M. Ab Initio
Calculations on the Defect Structure of $\beta$ Ga203. Phys. Rev. B 2013, 87 (23), 235206. https://doi.org/10.1103/PhysRevB.87.235206.

52. Ingebrigtsen, M. E.; Kuznetsov, A. Yu.; Svensson, B. G.; Alfieri, G.; Mihaila, A.; Badstübner, U.; Perron, A.; Vines, L.; Varley, J. B. Impact of Proton Irradiation on Conductivity and Deep Level Defects in $\beta-G a 2 O 3$. APL Materials 2018, 7 (2),

022510

https://doi.org/10.1063/1.5054826.

53. Hautier, G.; Miglio, A.; Ceder, G.; Rignanese, G.M.; Gonze, $X$. Identification and Design Principles of Low Hole Effective Mass P-Type Transparent Conducting Oxides. Nat Commun 2013, 4 , 2292. https://doi.org/10.1038/ncomms3292.

54. Zhang, K. H. L.; Xi, K.; Blamire, M. G.; Egdell, R. G. P-Type Transparent Conducting Oxides. J. Phys.: Condens. Matter 2016, 28 (38), 383002. https://doi.org/10.1088/09538984/28/38/383002.

55. Sasaki, K.; Higashiwaki, M.; Kuramata, A.; Masui, T.; Yamakoshi, S. MBE Grown Ga2O3 and Its Power Device Applications. Journal of Crystal Growth 2013, 378, 591-595. https://doi.org/10.1016/j.jcrysgro.2013.02.015.

56. He, H.; Orlando, R.; Blanco, M. A.; Pandey, R.; Amzallag, E.; Baraille, I.; Rérat, M. FirstPrinciples Study of the Structural, Electronic, and Optical Properties of $\mathrm{Ga}_{2} \mathrm{O}_{3}$ in Its Monoclinic and Hexagonal Phases. Phys. Rev. $B$ 2006, 74 (19),

195123. https://doi.org/10.1103/PhysRevB.74.195123.

57. Yamaguchi, K. First Principles Study on Electronic Structure of $\beta-G a 20$ 3. Solid State Communications - SOLID STATE COMMUN 2004, 131, 739-744. https://doi.org/10.1016/j.ssc.2004.07.030.

58. Brillouin zone and band structure of $\beta-G a 2 O 3$ Peelaers - 2015 - physica status solidi (b) - Wiley Online Library https://onlinelibrary.wiley.com/doi/full/10.100 2/pssb.201451551 (accessed May 22, 2019).

59. Varley, J. B.; Janotti, A.; Franchini, C.; Van de Walle, C. G. Role of Self-Trapping in Luminescence and \$p\$-Type Conductivity of Wide-Band-Gap Oxides. Phys. Rev. B 2012, 85 (8),

081109. https://doi.org/10.1103/PhysRevB.85.081109.

60. Mock, A.; Korlacki, R.; Briley, C.; Darakchieva, V.; Monemar, B.; Kumagai, Y.; Goto, K.; 
Higashiwaki, M.; Schubert, M. Band-to-Band Transitions, Selection Rules, Effective Mass and Exciton Binding Energy Parameters in Monoclinic \beta-Ga2O3. Phys. Rev. B 2017, 96 (24), 245205. https://doi.org/10.1103/PhysRevB.96.245205.

61. Yim, K.; Youn, Y.; Lee, M.; Yoo, D.; Lee, J.; Cho, S. H.; Han, S. Computational Discovery of P-Type Transparent Oxide Semiconductors Using Hydrogen Descriptor. npj Computational Materials 2018, 4 (1), 17. https://doi.org/10.1038/s41524-018-0073-z.

62. Perez-Tomas, A.; Mingorance, A.; Tanenbaum, D.; Lira-Cantu, M. Metal Oxides in Photovoltaics: All-Oxide, Ferroic, and Perovskite Solar Cells. In The Future of Semiconductor Oxides in NextGeneration Solar Cells; 2017. https://doi.org/10.1016/B978-0-12-8111659.00008-9.

63. Perez-Tomas, A., Functional Oxides for PhotoNeuromorphic Engineering: toward a Solar Brain. Advanced Materials Interfaces, 2019 (in press).

\section{Conflicts of interest}

There are no conflicts to declare. 